\title{
Low Luminosity Galaxies
}

\author{
J. I. Davies $^{\mathrm{A}, \mathrm{B}}$, S. Sabatini ${ }^{\mathrm{A}}$, and S. Roberts ${ }^{\mathrm{A}}$ \\ A School of Physics and Astronomy, Cardiff University, Cardiff CF24 3YB, UK \\ B Corresponding author. Email: jonathan.davies@astro.cf.ac.uk
}

Received 2004 May 3, accepted 2004 August 13

\begin{abstract}
Low luminosity (dwarf) galaxies play a crucial role in our current theories of galaxy and large scale structure formation. In the hierarchical picture they are the building blocks from which other structures form. These theories in their basic form overpredict the numbers of small dark matter halos (dwarf galaxies?) unless some form of star formation supression is invoked. In this paper we describe observations of dwarf galaxies in a range of different environments. We find that there are far too few dwarf galaxies in low density environments to be compatible with the theories. These observations are not consistent with an environmentindependent mechanism suppressing dwarf galaxy formation. It is also not clear how these mechanisms can supress star formation if dwarf galaxies have large mass-to-light ratios $(\approx 100)$. Either the whole idea of hierarchical galaxy formation has to be rejected or other environmentally dependent physical processes have to be invoked. We suggest that small, gas-rich dI galaxies have their evolution rapidly advanced as they move into the dense cluster environment.
\end{abstract}

Keywords: galaxies: dwarf — galaxies: clusters: Virgo cluster — galaxies: clusters: Ursa Major cluster — surveys

\section{Introduction}

Data from recent large redshift surveys carried out by SLOAN and $2 \mathrm{dF}$ have been used to define the global (averaged over all environments) luminosity function (LF) of galaxies (Blanton et al. 2001; Norberg et al. 2002). These two surveys produce a consistent result for the faintend slope of the LF, $\alpha \approx-1.2^{1}$. This value is somewhat flatter than typically predicted by most Cold Dark Matter (CDM) models of large scale structure and galaxy formation unless some form of dwarf galaxy formation supression is invoked (Mathis et al. 2002; Cole et al. 2000) - this is the sub-structure problem. Dwarf galaxies have been found in large numbers in a variety of rich, high density environments such as the Virgo cluster (Binggeli et al. 1985; Sabatini et al. 2003), Coma cluster (Milne \& Pritchet 2002), and Fornax cluster (Kambas et al. 2000) but in lower density environments very few have been discovered (Roberts et al. 2004). Ignoring the possibility of dark halos with no baryons at all, there are two possible interpretations consistent with the observations:

(a) CDM predictions are correct, but we need to invoke some additional mechanism that preserves primordial dwarf galaxies in some environments while destroying them in others;

(b) The dwarf galaxies found in rich clusters are a different population from the primordial ones predicted by CDM models, meaning that some physical process must have actually formed them solely in some environments.

\footnotetext{
${ }^{1}$ This is defined over a much brighter luminosity range than the one discussed in this paper.
}

Mechanisms proposed in support of the former hypothesis are:

(a) Squelching (Tully et al. 2002) - a suppression of dwarf galaxy formation in low density environments due to photoionisation occurring at a critical phase in the structure formation. Note though that the WMAP result now places the ionisation epoch at $z \approx 20$ (Spergel et al. 2003).

(b) Pressure confinement (Babul \& Rees 1992) - the pressure of the intra-cluster medium (ICM) reduces the gas loss produced by a feedback mechanism such as supernova-driven winds.

On the other hand, in support of the latter hypothesis, mechanisms that can form dwarf galaxies after the CDM dwarf galaxy formation epoch are:

(a) Tidal interactions (Okazaki \& Taniguchi 2000) galaxy-galaxy interactions and mergers can result in the formation of so-called tidal dwarfs.

(b) Harrassment (Moore et al. 1999) — infalling LSB disc galaxies in a cluster are subject to many high-speed encounters that can result in their morphological transformation into dwarf elliptical (dE) galaxies.

Trying to disentangle these issues requires a better understanding of how the mechanisms involved in galaxy evolution relate to the environment, and thus a detailed study of the properties of dwarf galaxies found in these different environments. In what follows we will try to analyse what makes a cluster different from the field or from a loose group, and which observables (galaxy numbers, colours, Hi content, velocity dispersion) can 


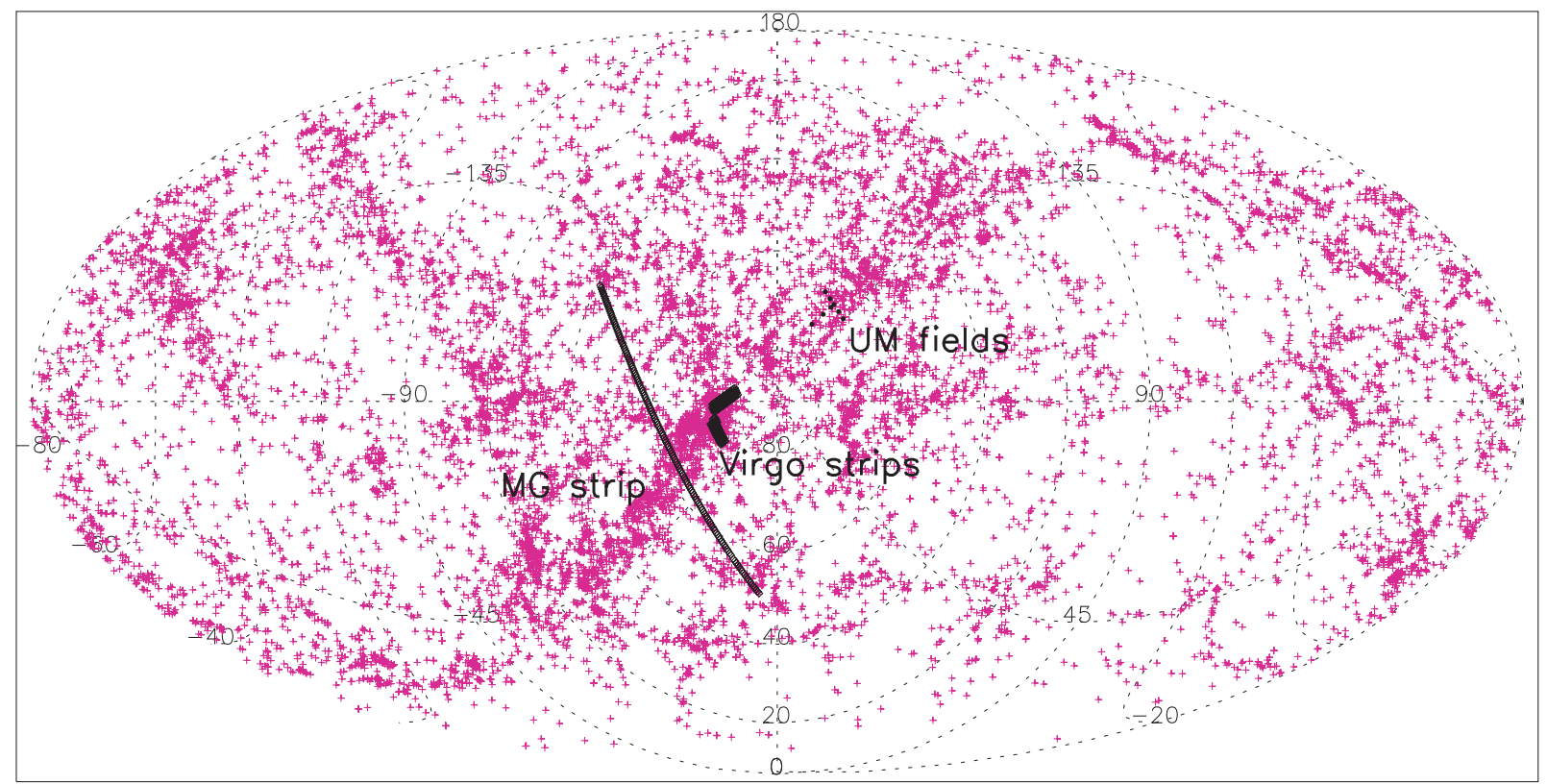

Figure 1 Positions of MGS, Virgo cluster data strips and fields in Ursa Major viewed from the North galactic pole. The MGS is indicated by the long thin line, it passes through the Virgo Southern extension at approximately its midpoint. The two Virgo data strips are situated above the MGS, whilst the UMa fields can be seen plotted as filled circles. Also plotted are all galaxies listed in NED with $v<4500 \mathrm{~km} \mathrm{~s}^{-1}$.

help distinguish between the two different interpretations given above.

\section{The Data Sets}

We have three data sets consisting of optical $B$ and $I$ data taken with the Wide Field Camera on the Isaac Newton Telescope and follow-up 21-cm data taken with the Arecibo Telescope.

\subsection{Millennium Galaxy Strip}

The Millennium Galaxy Strip (MGS) consists of an area of $30 \mathrm{deg}^{2}$ along the celestial equator (Liske et al. 2003). It extends through local regions of high and low galactic density. The strip begins in the Leo group, passing very close to NGC3521, before running through a relatively empty local area of space. The strip then passes through the Virgo Southern Extension, and back into a lower density region before ending in the higher density Virgo III cloud. Figure 1 illustrates the position of the data strip in relation to all galaxies listed in NED within $4500 \mathrm{~km} \mathrm{~s}^{-1}$.

\subsection{Virgo Cluster}

The Virgo cluster is an irregularly shaped, dense cluster of galaxies situated at a distance of approximately $16 \mathrm{Mpc}$ (Jerjen et al. 2003). It contains several hundred giant galaxies and a large population of dwarf galaxies (Binggeli et al. 1985). The cluster has a relatively short crossing time of approximately $0.1 H_{0}$ (Trentham \& Tully 2002). It is also an X-ray cluster and so in the cluster core, galaxies move through a hot inter-galactic gas (Young et al. 2002). We obtained data for two perpendicular strips extending outwards from the cluster centre (defined as M87) for $\approx 7 \mathrm{deg}$ (see Figure 1). The total area covered in the survey was $\approx 25 \mathrm{deg}^{2}$. Here we describe the results for the East-West strip $\approx 13 \mathrm{deg}^{2}$ (Sabatini et al. 2003).

\subsection{Ursa Major Cluster}

The Ursa Major cluster is a loose irregular cluster of predominantly spiral-type galaxies at approximately the same distance as Virgo (Trentham \& Tully 2002). It has a dynamical crossing time which is comparable to a Hubble time (Trentham \& Tully 2002), therefore is dynamically un-evolved with few galaxy-galaxy interactions having occurred. Our data fields are shown in Figure 1 in relation to the other data sets for the MGS and the Virgo cluster. The total area covered was $1.7 \mathrm{deg}^{2}$.

\section{$2.4 H_{I}$ Follow-Up Observations}

One of the greatest limitations to understanding the number density of dwarf galaxies has been the difficulty of obtaining distances (see for example, Jerjen et al. 2003). There are two reasons for this. The first is that many dwarf galaxies are not only of low total luminosity but also they have very low surface brightness - they are doubly cursed. This makes it extremely difficult to obtain an optical redshift. The second reason is that many dwarf galaxies (particularly in clusters) are apparently devoid of atomic gas, making a $21-\mathrm{cm}$ redshift impossible also. Even so, as part of this project we have used the Arecibo telescope to observe over 150 of our optical detections.

\subsection{Selection Criteria}

Our main objective is to compare the LSB dwarf galaxy population in different environments. To this end we need consistency in the types of objects we select. This is difficult if the types of objects in different environments are themselves very different. Current wisdom would 
describe the cluster population as dominated by rather featureless $\mathrm{dE}$ galaxies and the field by irregular dwarf galaxies (dI). Even so, to try and be as consistent as possible we have used the same selection criteria for each environment observed. These criteria (central surface brightness $23 \leq \mu_{0} \leq 26 B \mu$, exponential scale-length $3^{\prime \prime} \leq h \leq 9^{\prime \prime}$ ) were originally chosen following numerical simulations carried out by Sabatini et al. (2003). These criteria lead to a detection parameter space of $23 \leq \mu_{0} \leq 26 B \mu$ and $-10 \geq M_{B} \geq-14$ for the Virgo sample.

The above selection criteria and simulations were optimised for the detection of LSB dwarf galaxies at approximately the distance of the Virgo and Ursa Major clusters (Sabatini et al. 2003). For the MGS however, the data obtained is not all from an overdensity of galaxies concentrated at one distance. However, we still want to detect, for direct comparision, dwarf galaxies with the same intrinsic properties of magnitude and surface brightness as those in the Virgo cluster. The faintest galaxy $\left(M_{B}=-10\right)$ will, according to the surface brightness-magnitude relation $\left(\mu_{0} \approx 0.6 M_{B} \pm 0.1+32 \pm 1.3\right.$ ) of Driver (1999), have a scale size of $h \approx 3^{\prime \prime}$ at a distance of $21 \mathrm{Mpc}$. Thus, within this distance we expect to be able to detect all galaxies with intrinsic properties the same as those detected in the Virgo and Ursa Major cluster surveys using the same selection criteria. $^{2}$

\section{The Optical Detection Algorithm}

In order to investigate the possibility that previous surveys have missed a fainter population of dwarfs, we have applied an optimised Fourier convolution technique to our CCD images, detecting LSB dwarfs in the $B$ band down to surface brightnesses of $26 B \mu$ and $M_{B}=-10$ at the assumed Virgo cluster distance (see Sabatini et al. 2003 for full details).

\section{Results}

For comparison we define a dwarf-to-giant ratio (DGR) as the number of dwarfs with $-10 \geq M_{B} \geq-14$ and $23 \leq \mu_{0} \leq 26 B \mu$ divided by the number of galaxies with $M_{B} \leq-19$. In Table 1 we show how this relates to the more commonly used LF. Note the numbers in Table 1 are for galaxies of all surface brightnesses.

\subsection{The Virgo Cluster}

In the Virgo cluster we detected 257 galaxies which corresponds to an average 20 dwarf galaxies per $\operatorname{deg}^{2}$ (ranging from 40 at the centre to 4 at the cluster edge) and a DGR of about 20. Of these objects, 107 were observed at $21 \mathrm{~cm}$ with just three detections, all of which had very blue colours. The majority of optically detected galaxies have much redder colours (see below).

\footnotetext{
${ }^{2}$ The possiblity of missing some high surface brightness dwarf galaxies (see Drinkwater 2004) is discussed in Roberts et al. (2004).
}

Table 1. The Dwarf-to-Giant Ratio (DGR) relation to the luminosity function faint-end slope $(\alpha)$

\begin{tabular}{lr}
\hline$\alpha$ & DGR \\
\hline-1.0 & 4 \\
-1.2 & 18 \\
-1.4 & 80 \\
-1.6 & 367 \\
-1.8 & 1735 \\
-2.0 & 8371 \\
\hline
\end{tabular}

\subsection{The Millennium Galaxy Strip}

In the MGS we detected 110 objects, each of which were confirmed by eye. In the main, the detected objects are very different to those detected in our Virgo cluster survey. The Virgo cluster survey detections are predominately smooth diffuse objects (dE galaxies). In the field a large fraction of the detections are rather 'clumpy' objects and it is much more difficult to distinguish between what might be groups of faint distant objects from nearby irregular galaxies. For this reason we have divided our list of detections into two groups, those we are sure are individual galaxies (51 objects) and those that we are less confident of (59 objects). This corresponds to 1.7 and 3.7 galaxies per $\operatorname{deg}^{2}$ respectively. We have 21 optical redshifts and a further 18 detections at $21 \mathrm{~cm}$, from $56 \mathrm{HI}$ observations in total. In Roberts et al. (2004) we discuss extensively what we might be missing from this survey and, being as optimistic as possible, conclude that the DGR is at most 6 . The detected galaxies are generally more gas-rich than those in Virgo (all optical detections within $21 \mathrm{Mpc}$ also had 21-cm detections) and are generally blue in colour.

\subsection{The Ursa Major Cluster}

The detections correspond to about four objects per deg ${ }^{2}$ for Ursa Major, which is in reasonable agreement with the value obtained for the MGS data as a whole. The Ursa Major data is perfectly consistent with observations of the general field showing no enhancement, unlike the Virgo cluster, of dwarf galaxy numbers. There were no bright galaxies in any of the Ursa Major fields, because we only observed a small area, so we are not able to calculate a DGR for Ursa Major.

\subsection{Association with Bright Galaxies}

In the Virgo cluster we did not find any indication that the dwarf galaxies are associated with the giant galaxies. In the MGS data there is at most one galaxy with two dwarf companions. This is a far lower number of companions than might have been expected compared to the Milky Way. If the Milky Way was within $21 \mathrm{Mpc}$ we would expect five companions to be detected. We have checked that the area surrounding the bright galaxies was not masked during the detection phase leading to the removal of nearby companions (Sabatini et al. 2003). Why we are not finding a similar number of companions to these bright galaxies as 
Table 2. Mean values of $(B-I)$ colours for dwarf galaxies in different environments

\begin{tabular}{lccc}
\hline Environment & $\begin{array}{c}\text { Mass } \\
\left(10^{14} M_{\odot}\right)\end{array}$ & $\begin{array}{c}\text { Crossing time } \\
\left(H_{0}^{-1}\right)\end{array}$ & $(B-I)$ \\
\hline MGS & - & - & 1.0 \\
Ursa Major & 0.5 & 0.5 & 1.4 \\
Virgo & 8.9 & 0.1 & 1.5 \\
\hline
\end{tabular}

that found around the Milky Way is not at all clear and we are undertaking a more detailed study of the companions of nearby bright spiral galaxies.

\subsection{B - I Colours}

In Table 2 we give the mean values for the $(B-I)$ colours. There is a trend towards redder colours for richer environments. Data for Ursa Major is taken from Trentham et al. (2001).

\section{Discussion}

First let us summarise our results:

1. There are Virgo cluster dE galaxies that have a small range of colour which are preferentially found towards the centre of the cluster and are gas-poor. They are passively evolving (no or little star formation) and their colours are indicative of an older stellar population.

2. There are Virgo cluster dI galaxies which have a wide range of colours that tend to reside in the outskirts of the cluster. They appear to have star formation regions and the wide range of colours may reflect different current star formation states.

3. The MGS galaxies within $21 \mathrm{Mpc}$ are much bluer than those in the cluster and are all detected at $21 \mathrm{~cm}$.

4. There are about six times as many dwarf galaxies $\left(-14<M_{B}<-10\right)$ per giant galaxy $\left(M_{B}<-19\right)$ in the Virgo cluster than in the MGS. Because of this excess of dwarfs, the cluster cannot have been totally constructed from infalling galaxies or small groups like the Milky Way or Local Group.

5. Dwarf galaxies in the Local Group seem to be predominately associated with the bright galaxies. Virgo cluster dwarfs do not appear to be associated with the Virgo cluster bright galaxy population.

6. The luminosity function of the Virgo cluster over the range $\left(-14<M_{B}<-10\right)$ is much steeper than that in the MGS (much higher DGR). Also the HI mass function of the Virgo cluster appears to be less steep than the Hi mass function of the field (Davies et al. 2004). An obvious conclusion is that in the cluster gas has been more efficiently converted into stars.

7. In addition Conselice et al. (2003) have shown that dynamically the Virgo cluster $\mathrm{dE}$ poulation is similar to the spiral rather than the elliptical galaxy population. They argue that this implies the $\mathrm{dE}$ population is not primordial rather it is a population that has fallen into the cluster at a later date.
In the Sections below we briefly discuss possible physical processes acting on dwarf galaxies in the cluster environment and the influence they have on the results described above. Much of the analysis below is similar to that extensively and comprehensively discussed in a series of papers by Conselice et al. (2001, 2003). We formulate the problem in a slightly different way, concentrating on the mass-to-light ratios required to avoid gas stripping (see also Davies \& Phillipps 1989). A fuller description is given in Sabatini et al. (2004).

\subsection{Ram Pressure Stripping}

A cluster like Virgo has a substantial ICM which is detected via its X-ray emission (Vollmer et al. 2001). A galaxy moving through the ICM is subject to a ram pressure that can strip its gas. Following Gunn \& Gott (1972) and Davies \& Phillips (1989), we can set a limit for the dynamical mass-to-light ratio $M_{\mathrm{dyn}} / L_{B}$ that a galaxy requires to survive the ram pressure stripping:

$$
\left(\frac{M_{\text {dyn }}}{L_{B}}\right)>\frac{\rho_{\mathrm{ICM}} \times v_{\text {ort }}^{2}}{\sigma_{\mathrm{gal}}} 10^{0.4(\Sigma-26.8)}
$$

where $v_{\text {ort }}$ is the velocity of the galaxy through the ICM $\left(\mathrm{km} \mathrm{s}^{-1}\right), \sigma_{\mathrm{gal}}$ is the gas surface density in the galaxy $\left(M_{\odot}\right.$ $\mathrm{pc}^{-2}$ ), $\Sigma$ is the galaxy's surface brightness (blue mag/ $\operatorname{arcsec}^{2}$ ), and $\rho_{\mathrm{ICM}}$ is the density of the ICM $\left(\right.$ atom $\mathrm{cm}^{-3}$ ). This latter value depends on position in the cluster and can be described according to a $\beta$-model (Cavaliere \& Fusco-Femiano 1976) as follows:

$$
\rho_{\mathrm{ICM}}(r)=\rho_{0}\left(1+\frac{r^{2}}{r_{\mathrm{c}}^{2}}\right)^{-(3 / 2) \beta}
$$

where for the Virgo Cluster $\beta=0.45, \rho_{0}=4 \times 10^{-2} \mathrm{~cm}^{-3}$, and $r_{\mathrm{c}}=13.4 \mathrm{kpc}$ (Vollmer et al. 2001).

Combining Eqns (1) with (2) and assuming $v_{\text {ort }}=650 \mathrm{~km} \mathrm{~s}^{-1}$ (Conselice et al. 2001), i.e. the velocity dispersion of dwarfs in the Virgo Cluster, and $\sigma_{\text {gal }}=3 M_{\odot}$ $\mathrm{pc}^{-2}\left(10^{20}\right.$ atoms $\mathrm{cm}^{-2}$, the minimum Hi column density of star forming galaxies, see Minchin et al. 2003), we can obtain limits for the mass-to-light ratio for different values of surface brightness $(\Sigma)$ as a function of distance from the cluster centre. We conclude (see Sabatini et al. 2004) that dwarf galaxies are only stripped of their gas if they enter the core region and then only if they have mass-to-light ratios less than about 30 .

Recent work by Kleyna et al. (2002) on the velocity dispersions of stars in the outskirts of the Local Group galaxy Draco has revised its mass-to-light ratio from about 100 to 440. With mass-to-light ratios this high the dwarf galaxy population would be highly resistent to ram pressure stripping unless they pass deep into the cluster core.

There are however good reasons for believing that none of our detected dwarfs are actually in the cluster core at all. Dwarf galaxies within the core are subject to tidal forces that will pull them apart. We can estimate the size $(r)$ of a 
dwarf galaxy that would have a Roche limit at the cluster core radius.

$$
r=R_{\mathrm{c}}\left(M_{\text {dwarf }} / 3 M_{\text {cluster }}\right)^{1 / 3}
$$

where $R_{\mathrm{c}}$ is the cluster core radius ( $=0.5 \mathrm{Mpc}$ for Virgo), $M_{\text {dwarf }}$ is the mass of the dwarf galaxy $\left(\approx 10^{8} M_{\odot}\right)$, and $M_{\text {cluster }}$ is the mass within $R_{\mathrm{c}}\left(\approx 10^{14} M_{\odot}\right)$. This leads to a size of order $3 \mathrm{kpc}$, very similar to the sizes of our dwarf galaxies $(1 \mathrm{kpc} \approx 13$ arc sec at the distance of the Virgo cluster). We conclude that dwarf galaxies within the core will be severely disrupted by tidal forces (producing intra-cluster light, stars and planetary nebulae). Given that most of our galaxies in the projected core region are rather spherical dEs, we conclude that it is unlikely that they are actually in the core. We conclude that ram pressure stripping does not have a strong influence on our sample of Virgo cluster dwarf galaxies.

\subsection{Tidal Interactions}

Gravitational interactions can cause mergers, draw out discs and/or trigger starbursts. The mean colours and dynamical timescales shown in Table 2 indicate a dependence of galaxy properties with the cluster crossing time. The crossing time is related to the density of the cluster (not its total mass) with the most dense clusters having the shortest crossing times. The importance of interactions with other galaxies depends on the number density of galaxies $(n)$, the interaction cross-section $(\sigma)$, the velocity of the galaxy through the cluster $\left(v_{\text {ort }}\right)$, and the age of the cluster $(t)$. Using a simple rate argument and the total number of galaxies, virial radius and velocity dispersion for the Ursa Major and Virgo Clusters given in Tully et al. (1996), we find that the number of encounters experienced by galaxies in the Virgo Cluster is about 20 times higher than in the Ursa Major cluster. What the above does not tell us is how likely it is that a dwarf galaxy will have an interaction capable of completely disrupting and/or stripping its gas from it. To estimate this we can calculate the impact parameter as the square of the Roche limit distance.

$$
\sigma \approx r^{2}\left(3 M_{\text {gal }} / M_{\text {dwarf }}\right)^{2 / 3}
$$

where $M_{\text {gal }}$ is the mass of the galaxy the dwarf is interacting with. For a given cluster the number of interactions depends only on the cross-section $\sigma$. It is straight forward to show that a $10^{10} M_{\odot}$ galaxy is about 30 times more likely to interact in this way with another $10^{10} M_{\odot}$ galaxy than it is with a $10^{8} M_{\odot}$ galaxy. The reason is that the dwarf galaxy, because of its small size has to be very close to the large galaxy $(<10 \mathrm{kpc})$ for severe tidal disruption to occur (cf. the Sagattarius dwarf galaxy of the Local Group). This would be a problem if most of the Virgo dwarf galaxy population were closely associated with the giant galaxy population, but they are not. As we do not see large numbers of large galaxies undergoing disruptive tidal interactions we have to conclude that dwarf galaxy disruptive interactions are also uncommon. This is not a viable method of gas loss.
If the cluster dwarf galaxy population orginated from a tidally truncated population of larger field galaxies then their tidal radii should be $\approx R_{\mathrm{c}}\left(\sigma_{\text {dwarf }} / \sigma_{\text {clust }}\right)$ where $\sigma_{\text {dwarf }}$ $\left(\approx 10 \mathrm{~km} \mathrm{~s}^{-1}\right)$ and $\sigma_{\text {clust }}\left(\approx 700 \mathrm{~km} \mathrm{~s}^{-1}\right)$ are the dwarf galaxy and cluster velocity dispersions respectively. The smallest calculated tidal radii is of order $7 \mathrm{kpc}$ larger than the dwarf galaxies in our sample. They cannot be tidally truncated large galaxies. This seems to rule out the Moore et al. (1999) harrassment scenario as a means of producing the dwarf galaxies in our sample, but the infall of gas-rich dwarf irregulars like those detected by us in the outskirts of the cluster is still quite possible.

\subsection{Supernovae-Driven Winds}

In almost all CDM models supernova driven winds play a crucial role in supressing the continued star formation of dwarf galaxies. This cannot be the complete solution to the sub-structure problem, because of the large numbers of dwarfs found in some, but not all environments.

For a supernova-driven wind to expell the gas from a dwarf galaxy we require that the energy in the wind exceeds the gravitational binding energy or

$$
T_{\mathrm{W}}>10^{3}\left(M_{\mathrm{dyn}} / L\right) r 10^{0.4(26.8-\Sigma)}
$$

where $T_{\mathrm{W}}$ is the wind temperature and $r$ is the galaxies radius in kpc. For $\Sigma=26 B \mu$ and $r=1 \mathrm{kpc}, T_{\mathrm{W}}>$ $2 \times 10^{3}\left(M_{\text {dyn }} / L_{B}\right)$ for gas stripping to occur. Dekel \& Silk (1986) give a value of $T_{\mathrm{W}} \approx 10^{5} \mathrm{~K}$, leading to some uncertainty in whether this is a viable method at all for removing gas from dwarf galaxies if their mass-to-light ratios are large (Davies \& Phillipps 1989; see also Mac Low \& Ferrara 1999). This uncertainty is further compounded by the confining pressure of the ICM for cluster galaxies (Babul $\&$ Rees 1992). We conclude that supernova-driven winds are only a viable gas loss mechanism if dwarf galaxy massto-light ratios are low $(<100)$ and/or there are substantial star bursts and/or the pressure confinement is low.

\subsection{Gas Loss through Enhanced Star Formation in the Cluster Environment}

We believe that our observations can be entirely explained without the need for any of the gas stripping mechanisms described above. The gas that once was there is still there, but now in the form of stars, as witnessed by the very different cluster and field luminosity and Hi mass functions (Davies et al. 2004; Zwaan et al. 2003). We suggest that cluster dwarf galaxies have undergone accelerated evolution compared to the field because the cluster environment encourages the formation of stars.

Although we have shown that tidal interactions that strip a dwarf galaxy of its gas must be rare, numerical simulations do show that the several high-speed encounters that disc systems undergo in clusters can transform infalling galaxies into dEs (harrassment, Moore et al. 1999). The continually varying tidal forces compress gas, promoting enhanced star formation compared to dI galaxies in the field. Where we disagree with Moore et al. is that 
the origin of the $\mathrm{dE}$ population is infalling LSB disc galaxies. It is much more likely, we believe, to be the gas-rich dI galaxies observed on the cluster edge.

Can enhanced star formation alone explain the colour distribution of our galaxies and the lack of Hi emission from the dwarfs of our sample? If we assume the HI mass of a normal dwarf to be $10^{7} M_{\odot}$ and that each tidal interaction-triggered starbursts of $\sim 10^{8} \mathrm{yr}$ duration consumes $10^{6} M_{\odot}$ of gas (Leitherer \& Heckman 1995), then ten such starbursts can fully exhaust the Hi content of the dwarf. This would make dwarfs in clusters very different from isolated dwarf irregulars that typically have long gas depletion timescales of $\sim 20 \mathrm{Gyr}$ and which have experienced (and will continue to experience for at least another Hubble time) a slow, but constant, star-formation activity.

What would happen to the colours and absolute magnitudes of the dwarfs in our simple model of several starbursts?

According to Leitherer \& Heckman (1995) the change in $(B-I)$ colours in $10^{8}$ yr would be $\sim 1$ mag, regardless of metallicities and for either continuous star formation activity or a single starburst. The colour distribution of our sample is consistent with such a scenario. The cluster galaxies' average $(B-I)$ colour is about 1.5 , that is the typical colour for metal-poor globular clusters (Reed 1985).

\section{Conclusions}

CDM predicts that the first galaxies to form are dwarf galaxies in large over-densities. Is this consistent with our observations? We believe the answer is no for the following reasons, because the dwarfs

(a) are not more abundant in the field than in clusters.

(b) are not primordial in the sense that they formed in situ as the cluster formed - their velocity dispersion is closer to that of the spiral galaxy population (Conselice et al. 2003).

(c) do not come from the infall of units like the Local Group as we see them today — the dwarf-to-giant ratio of Virgo is too high.

(d) are not larger galaxies stripped of their gas or tidally transformed; they are too small.

(e) have not been created in tidal interactions of larger galaxies; these types of interaction are too rare.

We suggest that the dwarf galaxy population predominately arrived (are arriving) in the cluster as gas-rich dwarfs converting their gas into stars relatively rapidly and efficiently. It is not clear to us how this might be accommodated within the CDM model.

\section{References}

Babul, A., \& Rees, M. J. 1992, MNRAS, 255, 346

Binggeli, B., Sandage, A., \& Tammann, G. 1985, AJ, 90, 1681

Blanton, M. R., et al. 2001, AJ, 121, 2358

Cavaliere, A., \& Fusco-Femiano, R. 1976, A\&A, 49, 137

Cole, S., Lacey, C. G., Baugh, C. M., \& Frenk, C. S. 2000, MNRAS, 319,168

Conselice, J. C., Gallagher, J. S., \& Wyse, R. F. G. 2001, ApJ, 559

Conselice, J. C., O’Neil, K., Gallagher, J. S., \& Wyse, R. F. G. 2003, ApJ, 591, 167

Davies, J., \& Phillipps, S. 1989, ApSS, 157, 291

Davies, J., et al. 2004, MNRAS, 349, 922

Dekel, A., \& Silk, J. 1986, ApJ, 303, 39

Drinkwater, M. J. , et al., 2004, PASA, 21, 375

Driver, S. P. 1999, ApJ, 526, L69

Gunn, J. E., \& Gott, J. R. 1972, ApJ, 176, 1

Jerjen, H., Binggeli, B., \& Barazza, F. 2003, AJ, in press (astro-ph/ 0310779)

Kambas, A., Davies, J. I., Smith, R. M., Bianchi, S., \& Haynes, J. A. 2000, MNRAS, 120, 1316

Kleyna, J., Wilkinson, M. I., Evans, N. W., Gilmore, G., \& Frayn, C. 2002, MNRAS, 330, 792

Leitherer, C., \& Heckman, T. M. 1995, ApJ, 96, 9

Liske, J., Lemon, D. J., Driver, S. P., Cross, N. J. G., \& Couch, W. J. 2003, MNRAS, 344, 307

Mac Low, M. M., \& Ferrara, A. 1999, ApJ, 513, 142

Mathis, H., Lemson, G., Springel, V., Kauffmann, G., White, S. D. M., Eldar, A., \& Dekel, A. 2002, MNRAS, 333, 739

Milne, M. L., \& Pritchet, C. J. 2002, AAS, 201, 4211

Minchin, R., et al. 2003, MNRAS, 346, 787

Moore, B., Lake, G., Quinn, T., \& Stadel, J. 1999, MNRAS, 304, 465

Norberg, P., et al. 2002, MNRAS, 336, 907

Okazaki, T., \& Taniguchi, Y. 2000, ApJ, 543, 149

Reed, B. 1985, PASP, 97, 120

Roberts, S., Davies, J., Sabatini, S., van Driel, W., O’Neil, K., Baes, M., Linder, S., Smith, R., \& Evans, R. 2004, MNRAS, submitted

Sabatini, S., Davies, J., Scaramella, R., Smith, R., Baes, M., Linder, S. M., Roberts, S., \& Testa, V. 2003, MNRAS, 341, 981

Sabatini, S., et al. 2004, MNRAS, submitted

Spergel, D. N., et al. 2003, ApJSS, 148, 161

Trentham, N., Tully, B., \& Verheijen, M. A. W. 2001, MNRAS, 325, 385

Trentham, N., \& Tully, R. B. 2002, MNRAS, 335, 3

Tully, B. R., Somerville, R. S., Trentham, N., \& Verheijen, M. A. W. 2002, ApJ, 569, 573

Tully, B. R., Verheijen, M. A. W., Pierce, M. J., Huang, J. S., \& Wainscoat, R. J. 1996, AJ, 112, 2471

Vollmer, B., Cayatte, V., Balkowski, C., \& Duschl, W. J. 2001, ApJ, 561,708

Young, A., Wilson, A., \& Mundell, C. 2002, ApJ, 579, 560

Zwaan, M. A., et al. 2003, AJ, 125, 2842 